Comments on Paper by S. Penn and E.A. Martell, 'An Analysis of the Radioactive Fallout over North America in

Late September 1961'

\author{
By \\ Elmar R. Reiter
}

Technical Paper No. 57

Department of Atmospheric Science

Colorado State University

Fort Collins, Colorado

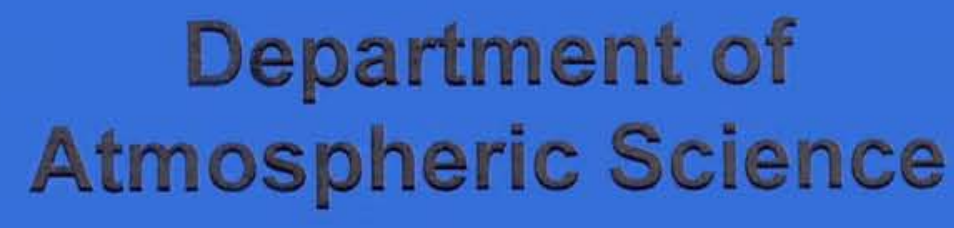

Paper No. 57 


\title{
Comments on Paper by S. Penn and E. A. Martell, 'An Analysis of the Radioactive Fallout over North America in Late September 1961'
}

\author{
ELMar R. REITER
}

\section{Colorado State University, Fort Collins}

In an interesting study Penn and Martell [1963] published several analyses which led them to the conclusion that the heavy radioactive fallout over widespread areas of the southeastern United States between September 17 and 22,1961, was at least in part produced by the vented underground shot in Nevada on September 15, 1961. While this paper certainly merits a broad and open-minded approach to the problem of radioactive debris transport, there are certain conclusions which are difficult to follow, especially in view of an investigation which I carried out on the same case [Reiter, 1963].

The trajectory which Penn and Martell computed backward from Flin Flon is in very good agreement with my findings; however, the forward extrapolation from September 15 to 17 , 1961 , is not. For some unspecified reason Penn and Martell consider the $2.5-\mathrm{km}$ level for their forward trajectory, instead of the isentropic level at which the debris was found, as they have done correctly for their backward trajectory.

As it turns out, the debris-carrying air masses underwent strong sinking motion associated with anticyclonic curvature after they had passed Flin Flon. This sinking motion actually carried the debris adiabatically into the mixing layer near the ground. Figures 1 and 2, taken from my report on this fallout case, show the "threedimensional' backward trajectories of the boundaries of the fallout regions $>100$ pe of September 17 and 18,1961 , computed along the $298^{\circ} \mathrm{K}$ potential temperature surface.

There should be no doubt that the debris encountered by balloon over Flin Flon actually did reach the southeastern United States, causing the abnormally high fallout intensity there. From the history of the Flin Flon debris, as concluded also by Penn and Martell, the Soviet atmospheric tests over Novaya Zemlya on September 10 will have to be made responsible for these high concentrations.
The fact that $\mathrm{I}^{181}$ (half-life 8.1 days) has been encountered in air samples of the debris should not necessarily be taken as an indication that the explosion had occurred close by. After all, the total travel time of the debris from Novaya Zemlya is comparable in magnitude with the half-life of this isotope; and we have no data about its original concentration. Radiation levels given by Anderson [1962] over Flin Flon for this particular case were about five orders of magnitude higher than were subsequently meas-

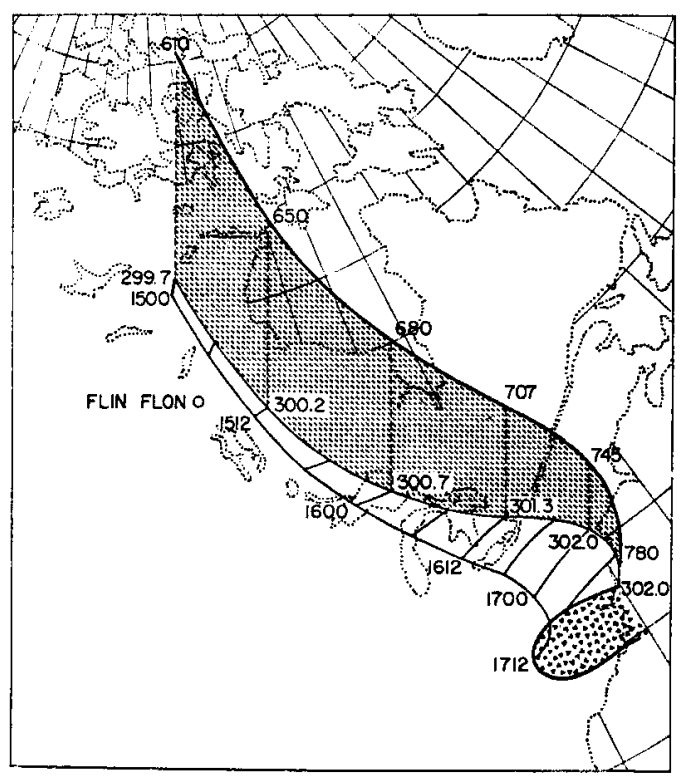

Fig. 1. Trajectories of the boundaries of the fallout area (irregularly shaded) observed on September 17, 1961, 1200 UT, over the United States east coast. The hatched region on top of the northernmost trajectory gives a three-dimensional view of this trajectory: its vertical extent indicates the movement of the particle along the vertical $p$ coordinate, the height of the hatched region being proportional to the pressure difference against the $800-\mathrm{mb}$ level; the numbers plotted are pressures $(\mathrm{mb})$, isentropic stream-function values $\left(10^{7} \mathrm{ergs} / \mathrm{g}\right)$, and map time of $298^{\circ} \mathrm{K}$ isentropic surface at these points along the trajectory. 


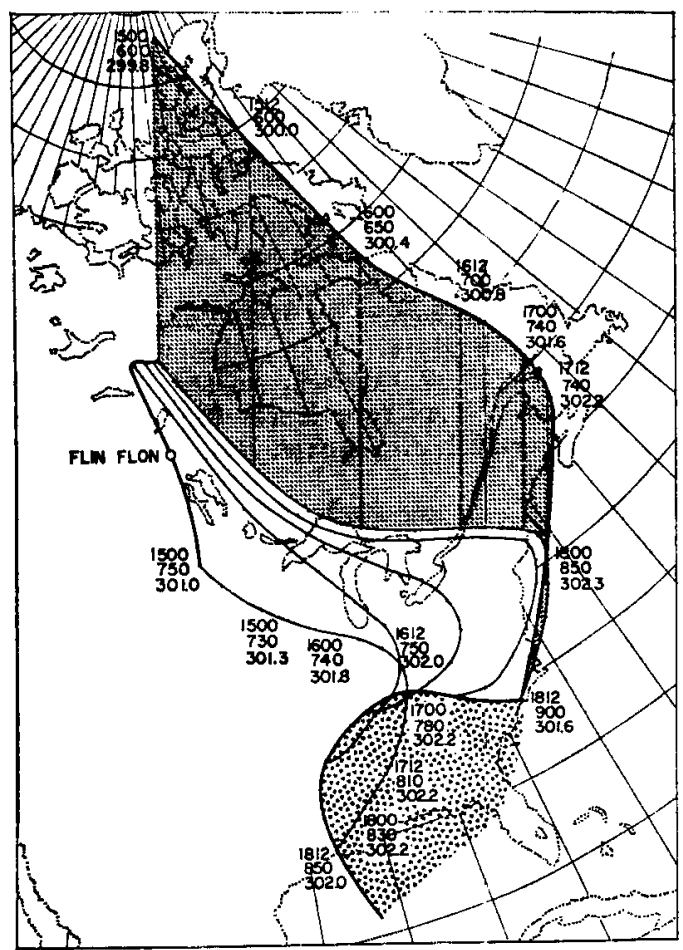

Fig. 2. Same as Figure 1, only for boundary of fallout region of September 18, 1200 UT. Map times, isentropic stream-function values, and pressures, again, are plotted along the 'three-dimensional' and the southernmost trajectories. Marks along the plane trajectories indicate 6-hour displacements.

ured on the ground over the southeastern United States. This, of course, could have other causes than the decay of short-lived products, namely (1) inadequate correspondence of ground-stationed air sampling equipment with balloonborne scintillation counters and (2) mixing of debris-carrying air masses with unpolluted air, especially within the friction layer near the ground.

In view of the facts stated above, it will be difficult to convince the reader that Nevada debris traveled to the east coast at the $12-\mathrm{km}$ level or the $343^{\circ} \mathrm{K}$ surface and then, by some precipitation process, was deposited at the ground. As was stated in my report, the fallout over the southeastern United States occurred within a pronounced high-pressure region with no precipitation over the largest part of the area. Some precipitation occurred along the fringes of the high-pressure region, associated with a frontal system advancing over the Middle West, and in the influence region of hurricane Esther advancing from the east. However, none of these precipitation areas affected the main fallout region to an extent that could either explain its shape or the maximum concentrations encountered. Precipitation measurements from New York, September 18 through 21; from Washington, D. C., September 19 through 21; and from Richmond, Va., September 19 through 21 , indicate above-average radiation values after the air sample measurements showed that the dry fallout pattern had already drifted off.

There is a conceivable possibility that these few occurrences of radioactive precipitation might have contained some Nevada debris. There is nothing else to support this assumption, however, than the statement that the $\mathrm{Ne}$ vada test had been a vented one. Unfortunately, we know nothing about the level at which this debris-if it was present at all-might have traveled. On the other hand, evidence from trajectory computations firmly supports the conclusion that the dry fallout in the region in question originated from the Soviet tests. This should easily explain why precipitation falling to the rear (i.e., to the northeast) of the main dry fallout zone shows high radiation levels. Condensation products wash out debris which is still being transported downward into the mixing layer along the general path shown in Figures 1 and 2, characteristic of the Flin Flon debris.

The fact that the fallout pattern near the ground changed shape, size, and intensity to a degree which cannot be explained by advection through low-level winds [Penn and Martell, 1963, Figure 5] is due to the rather extensive and strong sinking motion within the anticyclone which continuously brought contaminated air from the Flin Flon region into contact with the friction layer. No hypothetical precipitation effect is needed for an explanation of this phenomenon, and the statement of Penn and Martell that one should expect higher concentrations of debris on subsequent days to occur closer to the source region of this debris, if Soviet tests were to blame, apparently is based on an unrealistic model of exchange processes in the atmosphere.

Finally it should be stated that, while the dating methods used in establishing the age of 
the nuclear debris encountered in the atmosphere may not be as accurate as is desired, their results should not be ignored altogether. Instead of showing a forward shift in the average age of the debris, as the hypothetical Nevada debris from September 15 was mixed with debris of Soviet origin from September 10, and as should be concluded from Penn and Martell's assumptions, the contrary is observed. The mean age shifts from an estimated explosion date around September 10 to one around September 7 as the debris travels across the southeastern United States between September 17 and 21.

While Penn and Martell's suggestion that Nevada fallout is involved in the abnormally high level of radiation over the southeastern United States is interesting and certainly deserves to be studied, it lacks tangible evidence; in order to support it, evidence to the contrary will have to be overlooked or its validity questioned.

\section{REMERENCES}

Anderson, K. A., Thin atmospheric layers of radioactive debris during September 1961, Sci. Rept. to ONR, 23 pp., Cosmic Ray Group, Department of Physics, University of California, Berkeley, 1962.

Penn, S., and F. A. Martell, An analysis of the radioactive fallout over North America in late September 1961, J. Geophys. Res., 68(14), 41954207, 1963.

Reiter, E. R., A case study of radioactive fallout, Tech. Paper 42, 35 pp., Department of Atmospheric Science, Colorado State University, Fort Collins, 1963.

(Manuscript received August 23, 1963.) 